\title{
Complications following an unnecessary peri-operative plasma transfusion and literature review
}

\author{
Jay S. Raval ${ }^{1,2}$, Jonathan H. Waters ${ }^{3}$, Darrell J. Triulzi ${ }^{1,2}$, Mark H. Yazer ${ }^{1,2}$ \\ ${ }^{1}$ Department of Pathology, University of Pittsburgh Medical Center, ${ }^{2}$ The Institute for Transfusion Medicine, ${ }^{3}$ Department of \\ Anesthesiology, Magee Women's Hospital of University of Pittsburgh Medical Center, Pittsburgh, PA, USA
}

p-ISSN 1738-7949 / e-ISSN 2092-9129 http://dx.doi.org/10.5045/kjh.2012.47.4.298 Korean J Hematol 2012;47:298-301.

Received on April 19, 2012

Accepted on October 9, 2012

\section{Correspondence to}

Mark H. Yazer, M.D.

The Institute for Transfusion Medicine, University of Pittsburgh Medical Center, 3636 Blvd of the Allies, Pittsburgh, PA 15213, USA

Tel: + 1-412-209-7522

Fax: +1-412-209-7325

E-mail: myazer@itxm.org

(C) 2012 Korean Society of Hematology
Plasma is used to correct coagulopathies, but not all coagulation abnormalities are clinically significant enough to require correction before an invasive procedure. We report an 82 year old female who, in response to a mildly prolonged INR of unknown etiology, was unnecessarily transfused with plasma in advance of elective surgery. The patient suffered a moderately severe transfusion reaction, including hives and voice hoarseness, which caused a 4-week delay in her surgery. This delay and adverse reaction could have been avoided had the principles of evidence based plasma therapy, which we herein review, been followed and if the etiology of the mildly elevated INR been investigated before the day of her surgery.

Key Words Plasma, FFP, Transfusion, Allergic, Reaction, Complication

\section{INTRODUCTION}

Over 4 million units of plasma were transfused in 2007 in the United States [1]. The rate of plasma transfusion in the US per 1,000 population is more than twice that of other developed countries [2], leading some to suggest that it is being overused in the USA [3]. Plasma transfusion can be complicated by numerous different adverse events, including volume overload, transfusion related acute lung injury (TRALI) and allergic reactions. These latter reactions can demonstrate a spectrum of severity, ranging from mild urticarial skin rashes to anaphylactic shock. We report a case of a patient who had an unnecessary perioperative plasma transfusion and experienced an allergic reaction which resulted in a significant delay in her surgery, as well as review the literature on evidence based plasma therapy.

\section{CASE REPORT}

An 82-year-old female was scheduled to undergo elective knee replacement surgery secondary to degenerative joint disease. She had no personal or family history of a bleeding diathesis, nor was she on anticoagulation therapy. Pre-operative coagulation studies collected two weeks prior to the planned surgery date were slightly abnormal: prothrombin time (PT) $18.2 \mathrm{~s}$ (reference range, 11.7-15.3 s), INR 1.5 (reference range, 0.8-1.2), partial thromboplastin time (PTT) $39.4 \mathrm{~s}$ (reference range, 22.7-35.6 s). Her liver function tests and the parameters of her complete blood count from the same sample were all within the normal range. The cause of these coagulation abnormalities was not investigated, and repeat testing was not performed before the day of her surgery. Due to the mildly elevated INR, the patient's hematologist, who was medically managing this patient, recommended that 2 units of plasma should be transfused on the day of her surgery. After transfusion of the first unit of fresh frozen plasma, which was thawed at $37^{\circ} \mathrm{C}$ as per standard blood bank protocol and immediately issued for transfusion, on the day of surgery the patient developed widespread hives, hoarseness of her voice, and the subjective sensation of throat closure. Her oxygen saturation was maintained on room air $\left(\mathrm{SpO}_{2} \geq 96 \%\right.$ throughout this episode), her vital signs including her blood pressure were stable (temperature $36.5 \mathrm{C}$, heart rate $55-75 / \mathrm{min}$, respiratory rate $18-20 / \mathrm{min}$, systolic blood pressure $130-140$ $\mathrm{mmHg}$ throughout this episode), and no abnormal breath

This is an Open Access article distributed under the terms of the Creative Commons Attribution Non-Commercial License (http://creativecommons.org/licenses/by-nc/3.0) which permits unrestricted non-commercial use, distribution, and reproduction in any medium, provided the original work is properly cited. 
sounds were appreciated. Once this reaction was identified by the nurses in the pre-operative holding area, the unit physician stopped the plasma transfusion, and $25 \mathrm{mg}$ diphenhydramine, $20 \mathrm{mg}$ famotidine, and $8 \mathrm{mg}$ dexamethasone were administered. No other resuscitative measures were required. Within 2 hours all of these signs and symptoms had resolved. The blood bank investigation for hemolysis, which included a direct antiglobulin test, an inspection of her plasma for free hemoglobin, and a clerical check to ensure the correct unit was issued to the intended recipient, was negative. The transfusion medicine service was consulted and diagnosed this constellation of signs and symptoms as a mild-to-moderate allergic reaction. Due to this transfusion reaction, her surgery was cancelled and rescheduled for the next available operating room time which was 4 weeks later.

Two weeks before her surgery was reattempted, pre-operative blood tests again revealed a very slightly elevated INR (1.3) and the cause of this mild abnormality was not investigated. After being made aware of this INR value, the hematologist ordered 1 unit of plasma to be transfused immediately before the surgery. However, due to the patient's previous reaction to plasma, a thromboelastogram (TEG) analysis was performed immediately before the surgery began. Its values were within normal limits, plasma was not transfused, and the surgery was successfully completed utilizing spinal anesthesia with only $75 \mathrm{~mL}$ of blood loss. Vitamin K was not administered. The patient was discharged on the second post-operative day.

\section{DISCUSSION}

The main indication for plasma administration, apart from therapeutic apheresis procedures, is the correction of a clinically significant coagulopathy in a bleeding or peri-operative patient, such as that caused by oral anticoagulation or liver disease. However, not all prolonged coagulation parameters are clinically significant and require plasma transfusion. Thus there exists a disconnection between in vitro coagulation testing and clinical hemostasis. The PT and its calculated derivative, the INR, measure coagulation via the extrinsic pathway that contains factors I, II, V, VII, and X (including three of the four vitamin $\mathrm{K}$ dependent coagulation proteins). When the INR exceeds 1.6 , clotting factor levels begin to decrease below $0.30 \mathrm{IU} / \mathrm{mL}$ $(30 \%)[4,5]$. When the concentration of certain clotting factors are below $30 \%$, coagulation becomes impaired [6], and a plasma transfusion would be indicated to raise the coagulation factor levels, hence the commonly employed plasma transfusion threshold of INR $\geq 1.6$. When used appropriately, the hemostatic benefits of plasma outweigh the risks associated with its use which include volume overload, TRALI, and severe allergic reactions. The therapeutic effect of plasma lasts for approximately 8 hours [7], thus if it is to be used to reverse a coagulopathy in the peri-operative period then it should be administered as close to the surgical time as possible without necessitating a rapid infusion rate which could cause circulatory overload in the recipient.

In the case reported here, neither of the pre-operative INRs (1.5 and 1.3) met the plasma transfusion threshold. The literature indicates that patients with mildly abnormal coagulation tests do not experience significant peri-operative bleeding and that plasma is ineffective in correcting mildly abnormal test results [8-10]. A recent review of 14 studies (4 prospective, 8 retrospective, 1 mixed study design, and 1 in which the study design was unknown) compared bleeding outcomes after various bedside and interventional radiological procedures in patients with and without coagulopathies [8]. Though the definition of a coagulopathy varied between these studies (mean PTs as prolonged as $23 \mathrm{~s}$ and mean INRs as elevated as 1.73), the results demonstrated that there was no statistically significant difference in major bleeding between the two groups of recipients undergoing the same procedure. The authors concluded that slightly prolonged coagulation tests do not predict the occurrence of excessive bleeding after an invasive procedure [8]. The pre-surgical INRs of the patient in this report indicate that she was not at increased risk of bleeding and thus plasma therapy was not indicated. In another review of 12 randomized controlled trials of surgical patients where bleeding outcomes in patients who either received fresh frozen plasma (FFP) or a colloid volume expander were evaluated, the FFP intervention generally did not reduce blood loss or transfusion requirements [10]. The topic of clinical plasma transfusion has been recently reviewed $[11,12]$.

Furthermore, although counter-intuitive, patients with mildly prolonged INRs tend not to correct their abnormality when transfused with plasma. One large clinical study looked at the effect of FFP when transfused to patients with mildly elevated INRs (1.1-1.85) [9]. Of the 121 patients who had INRs in the above mentioned range and received FFP, only 1 of them normalized their INR (to $<1.1$ ) within 8 hours and only $15 \%$ of patients had correction in their INRs halfway to normal. The median decrease in INR after plasma transfusion was a mere 0.07 . In fact, there was no statistically significant difference in the decrease in the PT/INR between patients that received 1 versus 2 units of FFP, nor was there any difference in the decrease in the INR between patients with pre-transfusion INRs of 1.1-1.5 versus $1.5-1.85$. The authors concluded that transfusion of plasma does not correct mildly elevated INRs [9]. Thus given the INRs of the patient in this case report, the 1 or 2 plasma units that were ordered would not have had a hemostatic benefit, and indeed she experienced an adverse event due to its unnecessary administration.

One explanation for the inability of plasma to correct a mildly elevated INR stems from the relationship between the INR and coagulation factor levels. The experience with patients with hemophilia A or B (deficiency of factors VIII or IX, respectively) has demonstrated that coagulation becomes deranged when these and other factors decrease below 
$30 \%$ [6]. These factor concentration levels correspond to an INR of approximately $1.6[4,5]$. This means that a patient can have a modest elevation in their INR even though adequate factor levels are present for normal hemostasis. When the INR $<1.6$ there is an exponential relationship between it and the coagulation factor levels; however, as the INR exceeds 2.0, the relationship becomes linear meaning that a patient with an INR in this range would be expected to demonstrate a more rapid correction for every unit of plasma transfused compared to one whose INR is lower $(\leq 1.5)$ [5]. Thus the best predictor of the response to plasma transfusion is the pre-transfusion INR [13]; the closer it is to normal, the more miniscule the expected improvement will be after plasma transfusion. Furthermore, although plasma is prepared from healthy blood donors, almost $15 \%$ of the plasma units have an INR $\geq 1.2$ which is yet another reason why plasma is not effective in reversing a mildly elevated INR [14]. Lastly, in a stable $70 \mathrm{~kg}$ recipient with a 3000 $\mathrm{mL}$ plasma volume, transfusing 1 or 2 units of plasma (225$450 \mathrm{~mL}$ ) would increase the factor levels by $7.5 \%$ and $15 \%$, respectively which would not be expected to significantly reduce even a modestly elevated INR.

It is unclear why the etiology of the patient's INR of 1.5 was not investigated when it was first discovered 2 weeks before her initial surgery date. Although not elevated enough to require plasma therapy, it was an unexpected and abnormal result and its cause might have also had ramifications on her suitability for surgery. However, given that the mildly prolonged INRs were discovered 2 weeks prior to both scheduled surgery dates, a trial of oral vitamin $\mathrm{K}$ followed by repeating the INR several days afterwards could have been a benign intervention to resolve her mild INR elevations and could have avoided the risks of plasma transfusion [15]. Patients with mildly prolonged INRs (1.31.6) will effect some degree of correction without plasma therapy by simply having their underlying diseases treated and receiving supportive care such as hydration or correction of a base deficit [13]. It is also uncertain why the hematologist caring for this patient ordered another plasma transfusion prior to her rescheduled surgery in response to an even lower INR of 1.3 , especially considering she had an adverse reaction following the first transfusion. Fortunately, the clinical team was aware of this patient's transfusion history and successfully avoided another unnecessary plasma transfusion by demonstrating normal hemostasis pre-operatively with a TEG. The incorporation of point of care testing to evaluate clinical hemostasis in surgical patients, such as with a TEG, has been found to be useful in determining whether to transfuse plasma products and platelets [16-18].

In conclusion, adherence to the evidence based institutional guidelines for plasma therapy would have prevented this unnecessary plasma transfusion and the adverse reaction that it caused, thereby avoiding the delay in her surgery. The current clinical evidence indicates that plasma transfusion is not required for patients with an INR $<1.6$ as it does not confer a hemostatic benefit to the patient, and only exposes them to all of its attendant risks.

\section{REFERENCES}

1. Advisory Committee on Blood Safety and Availability. The 2007 national blood collection and utilization survey report. Washington, DC: Department of Health \& Human Services, 2007. (Accessed July 1, 2011, at http://www.hhs.gov/ash/bloodsafety/2007nbcus_survey.pdf)

2. Cobain TJ, Vamvakas EC, Wells A, Titlestad K. A survey of the demographics of blood use. Transfus Med 2007;17:1-15.

3. Wallis JP, Dzik S. Is fresh frozen plasma overtransfused in the United States? Transfusion 2004;44:1674-5.

4. Wachtel S, Kiss JE, Triulzi DJ, Winkelstein AW, Bontempo FA. The relationship of prothrombin time (PT) to clotting factor levels and the transfusion "trigger" for fresh frozen plasma (FFP). Blood 1994;84(Suppl):abst 682.

5. Dzik WH. The James Blundell Award Lecture 2006: transfusion and the treatment of haemorrhage: past, present and future. Transfus Med 2007;17:367-74.

6. Kitchens CS, Alving BM, Kessler CM, eds. Consultative hemostasis and thrombosis. 2nd ed. Philadelphia, PA: Saunders Elsevier Inc, 2007.

7. Hambleton J, Wages D, Radu-Radulescu L, et al. Pharmacokinetic study of FFP photochemically treated with amotosalen (S-59) and UV light compared to FFP in healthy volunteers anticoagulated with warfarin. Transfusion 2002;42:1302-7.

8. Segal JB, Dzik WH. Paucity of studies to support that abnormal coagulation test results predict bleeding in the setting of invasive procedures: an evidence-based review. Transfusion 2005;45: 1413-25.

9. Abdel-Wahab OI, Healy B, Dzik WH. Effect of fresh-frozen plasma transfusion on prothrombin time and bleeding in patients with mild coagulation abnormalities. Transfusion 2006;46:127985.

10. Stanworth SJ, Brunskill SJ, Hyde CJ, McClelland DB, Murphy MF. Is fresh frozen plasma clinically effective? A systematic review of randomized controlled trials. Br J Haematol 2004;126:139-52.

11. Yazer MH. The how's and why's of evidence based plasma therapy. Korean J Hematol 2010;45:152-7.

12. Triulzi DJ. The art of plasma transfusion therapy. Transfusion 2006;46:1268-70.

13. Holland LL, Brooks JP. Toward rational fresh frozen plasma transfusion: The effect of plasma transfusion on coagulation test results. Am J Clin Pathol 2006;126:133-9.

14. Holland LL, Foster TM, Marlar RA, Brooks JP. Fresh frozen plasma is ineffective for correcting minimally elevated international normalized ratios. Transfusion 2005;45:1234-5.

15. Grobler C, Callum J, McCluskey SA. Reversal of vitamin K antagonists prior to urgent surgery. Can J Anaesth 2010;57:45867.

16. Ferraris VA, Ferraris SP, Saha SP, et al. Perioperative blood transfusion and blood conservation in cardiac surgery: the Society of Thoracic Surgeons and The Society of Cardiovascular Anesthesiologists clinical practice guideline. Ann Thorac Surg 2007; 83(Suppl 5):S27-86. 
17. Tuman KJ, Spiess BD, McCarthy RJ, Ivankovich AD. Effects of progressive blood loss on coagulation as measured by thrombelastography. Anesth Analg 1987;66:856-63.
18. Sharma SK, Vera RL, Stegall WC, Whitten CW. Management of a postpartum coagulopathy using thrombelastography. J Clin Anesth 1997;9:243-7. 